\title{
El outsourcing como estrategia de eficiencia: Tres estudios de Caso en Costa Rica
}

\section{The outsourcing as an Efficiente Strategy: Three Study Cases in Costa Rica}

\author{
Fabián Arce Soto ${ }^{1}$ \\ farcesoto@yahoo.com.mx
}

Luz Chacón León ${ }^{2}$

luzchacon1958@gmail.com

Carolina España Chavarría ${ }^{3}$

carolina.espana.chavarria@una.cr

\section{Resumen:}

Esta investigación cualitativa, con la modalidad de estudio de caso, propicia la comprensión teórica y práctica del outsourcing en la administración de recursos humanos. Entre sus objetivos están el análisis conceptual y características del objeto de estudio, identificar sus usos, riesgos y el proceso que conlleva su contratación en algunas empresas costarricenses. El abordaje temático no pretende aprobar o desaprobar el uso del outsourcing como herramienta de gestión empresarial, más bien, procura apoyar la comprensión del público lector sobre este tema. A nivel práctico, la investigación intenta exponer el uso del outsourcing en un contexto real de negocios, evidenciando tres casos

\footnotetext{
${ }^{1}$ Máster en Administración de Empresas. Académico, Universidad Nacional, Costa Rica.

${ }^{2}$ Máster en Economía. Académica, Universidad Nacional, Costa Rica.

${ }^{3}$ Doctora en Innovación Educativa. Académica, Universidad Nacional, Costa Rica .
} 
particulares en Costa Rica. Esto permitirá ver a través de la perspectiva de usuarios y usuarias cómo funciona el outsourcing, qué actividades son las que han subcontratado y cuáles se prefieren realizar con personal de planta. Entre los hallazgos más significativos del outsourcing están: a) Su ejecución en función de metas específicas de la organización y b) Su implementación bajo la responsabilidad de un equipo integrado por personas internas y externas a la organización que aseguren el buen manejo del proceso y un destacado involucramiento de la alta gerencia y las gerencias de cada una de las áreas relacionadas con el proceso de subcontratación.

Palabras claves: Outsourcing, empresa, contratación.

\section{Abstract:}

This qualitative research, in the form of study cases, leads to the theoretical and practical understanding of the outsourcing in human resource management. Among its objectives are the conceptual analysis and characteristics of the object of study, identifying its uses, risks, and the process involved in some Costa Rican companies hiring. The thematic approach does not intend to approve or disapprove the use of outsourcing as a business management tool, rather, seeks to support the understanding of readers on this topic. On a practical level the investigation tries to expose the use of outsourcing in a real business context, demonstrating three particular cases in Costa Rica. This allows to see through the perspective of the users how the outsourcing is valued, which activities are those that have outsourced and which are preferred to be with plant personnel. Among the most significant findings of outsourcing are: a) Its execution on the basis of specific goals of the organization, and b) its implementation under the responsibility of a team of individuals internal and external to the organization that will ensure the good management of the process and a prominent involvement of senior management and the managers of each of the areas concerned in the process of outsourcing.

Key words: Outsourcing, company, recruitment

\section{INTRODUCCIÓN}

El outsourcing se está convirtiendo en una práctica muy aceptada para el desarrollo empresarial en todo el mundo. Para este estudio en particular fueron tomados en cuenta tres casos de empresas 
Revista Internacional de Administración de Oficinas y Educación Comercial Número 3, Año 2017; ISSN: 1659-3464; pp. 63-89

doi: http://dx.doi.org/10.15359/respaldo.3-1.4

costarricenses de diferentes sectores de la economía: urbanístico, de distribución energética y de la industria química. En todas las anteriores, el outsourcing ha servido como una herramienta de gestión que permite mejorar la eficiencia operacional.

Las empresas, en muchas industrias, externalizan o subcontratan cada vez más productos o servicios en sus negocios, debido a que logran aumentar el rendimiento, la rentabilidad y el valor para los accionistas de la compañía.

El outsourcing se practica en todo tipo de mercados, pero principalmente se puede observar en el sector energético, los serviciosfinancieros, desarrollodetecnología, telecomunicaciones, productos y servicios industriales y construcción. En general, las gerencias de las organizaciones, sean ejecutivas, de nivel medio o de primera línea, son las encargadas de tomar la decisión de subcontratar algún bien o servicio o de realizarlo de forma directa con los recursos de la organización.

La subcontratación es un instrumento mediante el cual se pueden obtener muchos beneficios, razón por la cual las organizaciones deben definir, de antemano, sus necesidades, intereses y expectativas de forma clara y precisa para todas las partes implicadas, ya que de esto dependerá la efectividad del contrato $\mathrm{y}$, consecuentemente, la calidad del producto, servicio o proceso brindado por el grupo proveedor. De allí la importancia de que las organizaciones dispuestas a implementar el outsourcing como estrategia de gestión empresarial realicen un análisis cuidadoso de los oferentes disponibles en el mercado, ya que puede existir la posibilidad de que algunas de estas empresas no cumplan con las expectativas de la organización y el producto, servicio o proceso final no tenga la calidad esperada por la clientela final.

A continuación, se presenta el esquema capitular de la investigación realizada. El primer capítulo consiste en un análisis teórico, mediante el cual se analizará información en función del tema para generar un criterio general de la visión que se tiene acerca del outsourcing. 
Una vez que se disponga de la información necesaria para abordar el tema, esta se organiza de forma coherente y según la revisión bibliográfica desarrollada. Es en este apartado donde se busca evidenciar los conceptos y fundamentos claves para el efectivo abordaje del objeto de estudio

Seguido del marco teórico, y con una visión general del panorama que presenta el outsourcing dentro de la administración de recursos humanos, se plantea un segundo capítulo, el cual consiste en la descripción del diseño de la investigación. En este bloque se muestran las decisiones metodológicas tomadas a la luz de los objetivos propuestos.

Como tercer capítulo se desarrolla un análisis descriptivo de tres empresas que utilizan el outsourcing, sobre una -o varias- funciones de su operación diaria. Para su desarrollo fue necesario contactar a uno o una de sus gerentes, en busca de la información necesaria para diagnosticar la empresa a la luz de la fundamentación teórica que sirvió de base para el estudio.

Por último, se desarrolla el cuarto capítulo del estudio dedicado a evidenciar las conclusiones y recomendaciones de todo el proceso de indagación, así como nuevas ideas respecto a la relación entre la teoría con la práctica del outsourcing en la administración de los recursos humanos.

\section{JUSTIFICACIÓN}

Desde el año 2012, Costa Rica, junto a otros países de Latinoamérica, se posiciona entre los primeros lugares en la utilización de servicios tercerizados (outsourcing), según el estudio anual realizado por la consultora internacional Tholons y publicado por Rodríguez del semanario costarricense El Financiero el 16 de enero del 2013.

Entre las razones que impulsan al sector empresarial a optar por el outsourcing se encuentran los ahorros en costos, eficiencia y el 
aprovechamiento de la valiosa experiencia específica en algunas actividades de individuos y empresas fuera de la organización. Durante estos procesos, las decisiones estratégicas se toman a la luz de la calidad del servicio que estas compañías están dispuestas a brindar.

Mediante la correcta aplicación de programas de outsourcing se pueden mejorar la calidad del servicio, la eficiencia en la utilización de los recursos y orientar los esfuerzos a las actividades fundamentales de la organización y a la razón de ser de esta misma. El crecimiento acelerado de los mercados y la demanda, cada vez mayor, por ser organizaciones más competitivas convierten al outsourcing en una herramienta que permite cambiar y evolucionar con mayor rapidez que si se dedicaran a realizar actividades que no son trascendentales o que, bien, son de apoyo a la gestión gerencial.

Antes de tomar alguna decisión relacionada con subcontrataciones, es fundamental determinar cuáles son las funciones que realmente se deben subcontratar y cuáles definitivamente no, ya sea porque son la fuente de las ventajas competitivas o porque los beneficios actuales superan en gran medida los costos de inversión en dichas funciones; otras veces las tareas que no se subcontratan manejan información confidencial, la cual debe permanecer lejos del conocimiento de algún tercero.

\section{OBJETIVOS}

\section{Objetivo general}

Analizar el concepto y características del outsourcing para determinar su importancia en la gestión empresarial.

\section{Objetivos específicos}

- Definir el concepto y las principales características del outsourcing. 
- Determinar los usos y riesgos del outsourcing.

- Identificar el proceso que conlleva una contratación por outsourcing en algunas empresas costarricenses.

\section{Marco teórico}

En este apartado se tratan aspectos teóricos relacionados con el objeto de estudio, a saber: la empresa, la administración, la estrategia y el proceso de outsourcing como recurso estratégico dentro de la gestión empresarial y organizacional.

\section{La gestión estratégica en las organizaciones}

Estas son épocas de turbulencias y cambios en todos los sentidos de la vida social y económica. La empresa, inmersa en esta vorágine, se encuentra obligada a elevar la eficacia y eficiencia de sus procesos productivos, la optimización de los recursos y la disminución de costos. Según indica Méndez (2012), la empresa es una forma de organización humana que cumple con diferentes funciones, características y actividades en alguno de los sectores económicos dentro de un sistema de reproducción. Cleri (2007) agrega que la mayor parte de la riqueza que se crea en las economías descansa en la empresa.

En este trabajo se entiende el concepto de empresa como aquella organización social, estructurada de tal forma que sus funciones y actividades se establezcan, coordinen y comuniquen para alcanzar los objetivos planteados. Según Chacón (2011), la empresa es la encargada de transformar materias primas (inputs) en productos o bienes y servicios (outputs).

Sin importar la clasificación de las empresas, sus actividades se circunscriben, en su parte medular, a planificar, organizar, coordinar, ejecutar y, controlar y evaluar. Su objetivo es maximizar la rentabilidad, la utilidad o el beneficio para garantizar crecimiento 
y sostenibilidad en el tiempo; sujeta a restricciones tecnológicas, de inversión y de las condiciones de mercado (Méndez, 2012).

De lo anterior se desprende la relevancia de la gestión empresarial como el coadyuvante teórico para lograr la sostenibilidad de las empresas. La gestión o administración de empresas, según Koontz, Weihrich y Cannice (2012), es el proceso mediante el cual se diseñan y mantienen acciones coordinadas, las cuales de forma individual no se lograrían.

Por tanto, para alcanzar los objetivos de una empresa, se requiere de distintas colaboradoras y colaboradores dedicados a diferentes actividades, entre ellas, al diseño de estrategias y su implementación, producción, ventas y demás. Esto implica la participación activa de un equipo de profesionales y personas en unión con las herramientas de gestión requeridas para garantizar la sostenibilidad empresarial anhelada.

Por otro lado, la administración estratégica, según Hofer y Schendel (2014), está basada en la unión de principios básicos como medio para alcanzar los objetivos, aunado con las políticas, y de ahí surge la estrategia como uno de los factores claves en el proceso de administración.

En la misma línea de los autores mencionados, la administración estratégica se centra alrededor de los siguientes aspectos fundamentales:

i Los objetivos claramente definidos y establecidos.

ii La formulación de la estrategia

iii Su implementación

iv Cambios en el entorno de la empresa.

En este marco surge, entonces, dentro de la gestión empresarial, la relevancia de vincular temas como el outsourcing como una metodología donde la estrategia empresarial puede apoyarse para alcanzar mayores niveles de rentabilidad. 


\section{Outsourcing: concepto y características}

El término outsourcing o subcontratación es definido de varias maneras. Para Mondy (2010), "la subcontratación es el proceso de contratar a un proveedor externo para que realice el trabajo que anteriormente se hacía dentro de la empresa" (p. 12). Durante décadas, la subcontratación ha sido una práctica utilizada por muchas organizaciones para asegurar el ahorro en los costos de producción u operación, mejorar la eficiencia y eficacia en la prestación de algunos servicios, entre otros. Esto resulta posible debido a algunas potencialidades del grupo proveedor, su alta especialización, experiencia, aprovechamiento de economías de escala y encuentro de sinergias.

El outsourcing actualmente se utiliza tanto en grandes corporaciones como en pequeñas y medianas empresas. Fórneas (2008) aclara que el concepto de outsourcing en ocasiones tiende a emplearse mal y detalla las siguientes características como necesarias para que una contratación se considere un outsourcing:

- Es un servicio que se contrata generalmente por más de un año, donde el sujeto contratado tiene un grado de autonomía significativo y adquiere algunas de las responsabilidades del contratante, además el contratado asume riesgos indirectos.

- Lleva asociados parámetros de calidad medibles y las condiciones son pactadas desde la primera contratación.

- Requiere de una medida de calidad objetiva. Esto implica que las actividades del sujeto contratado son controladas por el cliente, la responsabilidad del contratado es exclusivamente para la realización de tareas definidas. Si la contratación dura menos de un año, ya no se le podría considerar outsourcing. 


\section{Motivos para usar el outsourcing}

El mismo autor expone que las razones para la utilización del outsourcing; según el orden en que las considera importantes, son reducir el costo del servicio y la concentración de la empresa en las actividades primarias; la primera debido a que se pueden dar ineficiencias internas por no ser especialista en algunos servicios o por la falta de profesionales en ciertas áreas; y la segunda, dado que en algunas empresas los procesos de negocio son excesivamente complejos, por lo que se deben concentrar los esfuerzos en su actividad principal, lo que dificulta la gestión de otras áreas de apoyo.

Existen otras razones para recurrir al outsourcing relacionadas con la mejora en la calidad del servicio y el acceso a personal adecuadamente calificado. Esto último se puede atribuir a dos causas; por un lado es posible que se cuente con personal sobre calificado para realizar algunas tareas, situación que podría causar frustración y desmotivación, o bien que suceda lo contrario y no exista el personal con las competencias y la experticia requerida para desempeñar un puesto en particular. En cualquiera que sea el caso, lo importante es conformar equipos de trabajo en donde todos y todas desarrollen las competencias necesarias para la promoción de un servicio de calidad. Este se destacará por la simplificación de los procesos de negocio en donde se minimizan el error, los reprocesos y los costos de oportunidad, a la vez que permite la adición de nuevas líneas de negocio, la búsqueda de nuevos mercados o la innovación de productos.

El outsourcing también puede ser utilizado como herramienta para la reducción del tiempo que tarda un producto desde su creación hasta su introducción al mercado. Esto implica un destacado aprovechamiento de las tecnologías y el conocimiento de personas expertas en la materia. En algunos casos para las pequeñas y medianas empresas, tener este tipo de recursos de manera permanente es casi imposible, lo cual podría afectar su competitividad. 


\section{Riesgos del outsourcing}

Sobre los riesgos del outsourcing, Fórneas (2008) considera que pueden ser varios, como no alcanzar los objetivos planteados, ya sea en costos, calidad o en la satisfacción de las necesidades del personal interno. Estos riesgos pueden ser controlados a lo interno de la organización y otros por la empresa contratada. En cuanto a conflictos internos, el autor los enfoca desde la perspectiva de los grupos trabajadores que se preocupan por sus puestos o bien la inadaptación a la nueva situación, por lo que se requiere de un importante y continuo apoyo de la alta gerencia.

Otros riesgos a destacar son el grado de dependencia en que puede caer la empresa al decidir entregar la prestación de un bien o servicio a un tercero, el surgimiento de conflictos que se puedan presentar en términos de cumplimiento de contratos, o un inadecuado proceso de selección de entes proveedores en donde se podría dejar por fuera el análisis de la situación financiera del sujeto proveedor, su solvencia técnica, recomendaciones de clientela reconocida en el sector.

Sin duda alguna, uno de los principales riesgos que deben manejarse con mayor ahínco y prudencia es la posibilidad de pérdida de control o conocimiento sobre un bien o servicio. El autor recalca sobre la importancia, en estos casos, de su permanencia en la empresa, pues al poder externalizarle, es más probable que no desaparezca.

\section{Proceso del outsourcing}

El proceso de contratación del servicio de outsourcing es establecido por Fórneas (2008) de una manera bastante estructurada, que inicia con la identificación del servicio a externalizar, continúa con el establecimiento de objetivos a conseguir, así como las tareas necesarias para su consecución. Dentro de dichas tareas se encuentra la identificación de los sujetos 
proveedores, de los cuales se debe obtener la mayor información posible, la empresa que elige está en la potestad de solicitar información financiera, referencias del sector, perfil de la empresa y cualquier otra evidencia de solvencia económica y moral.

Posteriormente, se deben solicitar las propuestas de cada oferente para obtener el panorama económico más completo, teniendo claro que el costo del servicio no debe ser el único factor a considerar; de hecho, una propuesta con un precio mucho más bajo puede ser indicio de desesperación por parte del ente proveedor que no es comercialmente aceptado.

Como paso siguiente, se revisa el borrador del contrato a utilizar. Este debe contener los compromisos a exigir, así como los diferentes puntos por negociar. A continuación, se procede con la selección del líder del proceso y sus responsabilidades; así como de los individuos negociadores y sus competencias. Luego se establecen las condiciones que regirán el contrato.

\section{Outsourcing: costos y eficiencia}

El outsourcing favorece la disminución de costos a aquellos asociados a la operación y ejecución de funciones tales como: mano de obra, gestión de materiales, administración de sistemas de información, área de servicio al cliente, entre otros. También, se le considera una estrategia empresarial para el aprovechamiento de la estructura de inversión que otras empresas ya han realizado, de esta manera, permite reducir las inversiones propias, lo que libera recursos financieros que se podrían destinar a otra parte del proceso productivo o de la cadena de valor.

Otro aspecto importante de resaltar sobre el outsourcing es que le facilita, a la empresa contratante, asociar los costos a mediciones de productividad previamente establecidas. De esta forma, evita tener recursos ociosos que son muy comunes cuando la empresa se encuentra en etapas de baja actividad por disminución de la demanda. De allí que Schneider (2004) 
destaque, entre sus principales características, la especialización no intrínseca al núcleo de la organización contratante.

Otro impacto positivo en la eficiencia de la empresa consiste en alinear los recursos humanos y tecnológicos a las funciones y tareas estratégicas, más relacionadas con la naturaleza o razón de ser de la organización. Lo anterior, conlleva a un incremento potencial de la capacidad productiva de la empresa, ya que se pueden atender demandas extraordinarias del mercado sin aumentar la estructura o infraestructura.

Resulta evidente que el proceso de outsourcing, además de orientar los recursos hacia funciones más estratégicas; también abre las puertas a las mejores prácticas y tecnologías que otras empresas, con mayor experiencia o know how, poseen y se lo ofrecen a otras organizaciones.

En este sentido, el impacto en la eficiencia operativa de la empresa contratante es una mayor oferta de servicios, con más capacidad de respuesta a las demandas crecientes, mejor calidad en el servicio y la posibilidad de acceder a otros mercados, nacionales o internacionales.

Pero también, la subcontratación genera otro tipo de beneficios, tal vez más intangible, pero real en caso de concretarse, y es el establecimiento de una relación contractual que podría derivar en una relación más formal, como, por ejemplo, una sociedad, entre el contratante y la persona o empresa contratada.

\section{Marco metodológico}

La investigación inicia su diseño en el III cuatrimestre del año 2014, en el 2015 realiza su trabajo de campo y concluye con toda una incorporación de mejoras en el 2016. Para su diseño se recurrió al paradigma de investigación cualitativo, el cual pretende ahondar en la realidad de una situación, en este caso la implementación del outsourcing en la gestión empresarial. 
Con esta escogencia metodológica se aprende sobre la base de experiencias y situaciones de la vida real para construir un nuevo aprendizaje sobre una temática en particular y su incidencia en el entorno.

La descripción que se brinda del objeto de estudio, el outsourcing, es meramente narrativa a partir del análisis documental, la observación y la comunicación con responsables de la gestión empresarial, mediante la aplicación de una entrevista dirigida a la gerencia del área de Recursos Humanos. Este instrumento sirvió para la consulta de diversas temáticas relacionadas con el outsourcing, entre ellas: tipos, procesos y requisitos para la subcontratación, perfil de puesto de la persona encargada de las subcontrataciones, beneficios y desventajas de la subcontratación.

La posibilidad que brinda este tipo de abordaje investigativo permite el acceso a distintas opciones o soluciones, las cuales facilitan el pensamiento divergente desde un punto de vista multidimensional, orienta a la acción y se abre a la compresión de los hechos o conflictos en los diferentes escenarios de la vida laboral.

La población de estudio fue escogida de manera intencional. Contóconla participación detres casos deempresas costarricenses representantes de diferentes sectores de la economía: urbanístico, de distribución energética y de industria química. El criterio de inclusión que prevaleció para su escogencia fue el hecho de que en todas estas el outsourcing ha servido como una herramienta de gestión para la mejora de la eficiencia operacional.

La primera, una urbanizadora dedicada a la construcción de viviendas orientadas al mercado de clase media-alta, con más de 40 años de experiencia, en los que ha desarrollado más de 50 proyectos inmobiliarios en el país, posee certificaciones de calidad y han sido acreedores del Premio a la Excelencia de la Cámara de Industrias de Costa Rica durante varios años. La 
razón de escoger dicha empresa es la experiencia de utilizar servicios de outsourcing, para lo cual se realizó una entrevista a su gerente de Recursos Humanos.

La segunda empresa seleccionada fue la cooperativa Coopeguanacaste R.L., ubicada en la provincia de Guanacaste, Costa Rica, y dedicada a la distribución de energía eléctrica a los hogares, comercios e industria de la mayor parte de la zona de la Península de Nicoya. Cuenta con más de 61000 personas asociadas. Es una organización que desarrolla proyectos hidroeléctricos e investiga sobre otras fuentes de generación eléctrica con el objetivo de lograr un nivel de autosuficiencia energética aceptable a las condiciones climáticas actuales.

La escogencia de Coopeguanacaste radica en el uso que hace del outsourcing, su experiencia en el manejo del proceso y el impacto económico y ambiental que la organización ocasiona en la zona guanacasteca.

Se entrevistó al gerente de Generación y Proyectos, por ser el área que más contrataciones externas genera a la organización.

Por último, la tercera empresa escogida fue Sherwin-Williams. Esta es una productora de barnices y pinturas a nivel mundial. Cuenta con certificaciones mundiales que garantizan la calidad de los productos. Inicia operaciones en Costa Rica en el año 1968, lo anterior evidencia su impacto relativo en la economía costarricense. Además, también tiene experiencia en el manejo de los procesos de subcontratación.

Se entrevistó a la gerente de Recursos Humanos de Costa Rica.

\section{Análisis de resultados}

A continuación, se presenta el análisis de resultados obtenido del trabajo de campo, el cual inició en el III cuatrimestre del año 2014 y concluyó a finales del 2015. Cada uno de los casos se 
expone atendiendo a los objetivos propuestos para el desarrollo de la investigación, la cual culmina en su totalidad en el I cuatrimestre del año 2016.

\section{Caso uno: Empresa urbanizadora}

La persona entrevistada indica que la empresa utiliza los servicios de outsourcing desde hace 20 años y que el enfoque de la empresa es que toda tarea puede ser subcontratada, por lo que actualmente están ejecutando con esta modalidad los servicios de limpieza, mensajería, construcción de obras tanto urbanísticas como residenciales y presupuestos, auditoría y seguridad. Algunos servicios que ocasionalmente trabajan de esta forma son diseño y supervisión electromecánica.

Al consultar propiamente por el área de Recursos Humanos, se aclara que es propia y los procesos se ejecutan por el personal de la misma empresa, principalmente en cuanto a la dotación de personal; solo la parte de capacitación se realiza de forma subcontratada la mayor parte de las veces. Esto se debe a que la empresa considera que esta es un área muy sensible y que debe ejercerse por personas que conozcan a profundidad la organización.

\section{Aplicación del tema}

Aunque la Gerencia de Recursos Humanos es un área directamente involucrada en las decisiones de outsourcing, la subcontratación específicamente la coordinan con personas de cada área de especialización; así, lo que tiene que ver con construcción está a cargo del director de Ingeniería, los servicios relacionados con el área contable del gerente Financiero o bien los servicios administrativos se coordinan con la asistente de la Gerencia General.

El apoyo de la Gerencia General y de las Gerencias de las áreas respectivas es valioso también porque un aspecto clave es 
mantener contratos muy bien definidos y en orden, por lo que deben ser revisados por quienes conozcan directamente el servicio.

Por otro lado, aunque es personal externo, como parte de su responsabilidad se vela por contratar los servicios de empresas serias que sean cumplidas en la designación del personal; que respeten sus derechos y obligaciones; que no paguen menos del salario mínimo establecido para cada puesto y que les garanticen todos los beneficios y derechos.

Otro aspecto que se discute es que consideran un ahorro en costos importante, el cual permite mejorar la rentabilidad y, de igual forma, una mayor eficiencia en sus operaciones. La empresa procura mantener relaciones de largo plazo con el fin de involucrar al personal externo para que, aunque no trabaje en forma directa con la empresa, logre identificarse y "ponerse la camiseta de la compañía".

\section{Procedimiento}

En cuanto al procedimiento que se sigue, se inicia con el envío de un cartel de requisitos al menos a tres empresas especializadas en el área respectiva para que oferten y presenten requisitos tales como cartas de recomendación, referencias que se puedan consultar y el compromiso de presentación mensual del pago de planilla ante la CCSS para asegurar que esté debidamente asegurado.

Estos requisitos junto con las cotizaciones se presentan a la Gerencia General y se escoge la mejor oferta tomando en cuenta no solo que el precio se ajuste si no que se adapten a todas las necesidades de la empresa.

\section{Aspectos positivos e inconvenientes}

Un aspecto positivo de la subcontratación, y que resalta el entrevistado, es el hecho de que en caso de que falte algún sujeto trabajador perteneciente a la empresa que brinda el 
servicio de outsourcing, como, por ejemplo, un mensajero o un oficial de seguridad, simplemente se solicita su sustitución de manera inmediata, sin ninguna afectación en la operatividad de la organización.

Además, subcontratando estos servicios, la empresa dedica sus mayores esfuerzos de gestión a las actividades neurálgicas de la organización, lo que contribuye de manera significativa al logro de los objetivos estratégicos, dejando las áreas más operativas o de apoyo a las empresas que brindan los servicios de outsourcing.

En relación con los posibles inconvenientes del outsourcing, se mencionó que en definitiva se debe mantener una mayor supervisión de los trabajos que se ejecutan, así como mayores controles para el cumplimiento de las tareas, ya que, de darse un problema en el servicio contratado, al final quien debe dar la cara al cliente es la empresa que subcontrata, aunque el problema se origine a nivel externo.

Asimismo, otras posibles desventajas que se comentaron fueron la mayor rotación del personal asignado lo que, a su vez, ocasiona de alguna manera falta de identificación con la empresa; o bien, se ha experimentado en alguna ocasión incumplimiento entre lo ofrecido y lo entregado por el servicio, por lo que se requieren acciones inmediatas de corrección o de ruptura del contrato.

\section{Caso dos: Coopeguanacaste R.L.}

La persona entrevistada indica que la cooperativa cree en los trabajos por outsourcing y los beneficios que generan. Han apostado a las subcontrataciones en las diferentes áreas de la organización. Con relación al área de Desarrollo de Generación y Proyectos, las tareas sujetas a la subcontratación son: construcción de línea de transmisión, lectura de medidores, operación y mantenimiento de las plantas de generación, construcción de línea de telecomunicación, como las más importantes. 


\section{Aplicación del tema}

La cooperativa está dividida en cuatro áreas de negocio, a saber: generación, distribución y comercialización de energía eléctrica, y telecomunicaciones. Cada gerente de área maneja, de acuerdo con su estrategia, el tema de subcontrataciones.

Ante la pregunta de que si la subcontratación permite ahorros en los costos, la respuesta fue categórica en términos de que la experiencia les indica que, aunque cada contratación es diferente, en general hay una importante disminución en los costos y en los tiempos de ejecución de la obra.

Cuando se realizó la pregunta de opinión relacionada con la mayor eficiencia en los trabajos en los procesos de subcontratación, se indicó que la teoría es muy clara y abundante cuando se discute sobre este tema; no obstante, la experiencia con Coopeguanacaste demuestra que, para alcanzar niveles superiores de eficiencia, se requiere de mayor supervisión y llevar un control estricto sobre los indicadores de costos que el área financiera tiene establecidos. Resulta de vital importancia el papel que tiene la supervisión y el monitoreo en dichos indicadores.

También, el entrevistado relató que una de las razones por las que un departamento subcontrata es la liberalización de recursos humanos para dedicarlos a otras tareas primarias del negocio.

Cuando se elaboró la pregunta relacionada con qué otros aspectos se deben tomar en cuenta para decidir subcontratar o mantener internamente el proceso productivo, respondió que en orden de importancia, los ahorros en los costos de la actividad, y resaltó el impacto positivo en el negocio, cuando la subcontratación va de acuerdo con el plan estratégico.

Por último, respondió que, en el caso de Coopeguanacaste, el área de Recursos Humanos es propia y forma parte de la estructura de costos de la organización. 


\section{Procedimiento}

Dado que Coopeguanacaste es una figura jurídica de responsabilidad limitada, el proceso de outsourcing se realiza por medio de carteles de licitación privada. Según indica el entrevistado, los requisitos de cada uno de los carteles varían de acuerdo con el alcance que se quiere abarcar con la subcontratación. De este proceso se encarga el gerente que tenga la necesidad de subcontratar, por lo tanto, todos los carteles de licitación son diferentes en su estructura, contenidos y términos de referencia.

\section{Aspectos positivos e inconvenientes}

Cuando se generó la pregunta relacionada con la consideración de que el Departamento de Recursos Humanos, o al menos algunos procesos de esta área, debieran subcontratarse, su respuesta indicó que a criterio personal, algunos procesos como los de evaluación del desempeño y de reclutamiento y selección de personal es conveniente que se subcontraten para evitar la injerencia de posiciones personales a la hora de realizar una evaluación o selección de personal colaborador. También, agregó que la empresa Holcim de Costa Rica subcontrata algunos de los servicios mencionados.

Finalmente, señaló que el proceso de outsourcing podría tener posibles impactos negativos (o desventajas), cuando la empresa contratante tiene poca experiencia en este tipo de actividades, o que los seguimientos no sean los adecuados, lo cual puede conducir que más bien se incremente la ineficiencia o la burocracia.

\section{Caso tres: Sherwin-Williams}

En este caso, ante la pregunta planteada a la entrevistada sobre si la empresa subcontrata proveedores externos, indicó 
que al ser una transnacional se realizan procesos de análisis de la inversión y se relaciona con el presupuesto asignado para los rubros sujetos a subcontratarse. Las subcontrataciones más comunes son los procesos de capacitación, asistencia legal y fiscal, y la validación de la gestión del personal.

También indicó que no existe una persona responsable de los procesos de subcontratación, sino que cada área propone, cotiza y se reúne con los posibles proveedores. Cada subcontratación debe justificarse según una guía de planeamiento y presupuesto de las áreas.

\section{Aplicación del tema}

Con respecto a la pregunta relacionada con la consideración de que si la subcontratación permite un ahorro en costos para la empresa, la entrevistada mencionó que, más que un ahorro en costos, valida, desde una perspectiva externa, los procesos para los cuales son contratados. Esta validación, para la empresa, es muy importante ya que les permite alinear procedimientos y aumentar la eficiencia de las labores.

También reveló que los mayores niveles de eficiencia observados se derivan de la medición que se realiza de los resultados obtenidos, por ejemplo, cuando se subcontrata para evaluar el clima organizacional y, a partir de los resultados obtenidos, se genera un plan de acción para remediar los aspectos más álgidos del proceso interno organizacional.

De igual forma, cuando se planteó la pregunta de qué otras razones, además del ahorro y eficiencia, determinan la subcontratación, respondió que para la empresa la validación de procesos es un factor importante para decidir si se subcontrata o no. Otro elemento determinante es que analizan los costos relacionados con la subcontratación, los comparan y luego se toma la decisión. También, toman en cuenta el desarrollo interno del personal y las oportunidades de involucrarlo en proyectos específicos. 
El área de Recursos Humanos en la empresa Sherwin-Williams es propia y forma parte del staff de la organización.

La entrevistada considera que el Departamento de Recursos Humanos, o al menos algunos procesos de esta área, debieran subcontratarse, e indica que, desde su punto de vista, procesos como salud ocupacional, legislación laboral, aplicaciones específicas de herramientas, entre otros. Agrega que conoce empresas que utilizan estos servicios entre otros.

\section{Procedimiento}

En relación con el tema de los procesos que se siguen en Sherwin-Williams para la subcontratación, indicó que se realiza lo siguiente:

1. Tres cotizaciones.

2. Que las empresas o personas sujetas de ser subcontratadas posean experiencia en el área requerida.

3. Empresas o personas con condiciones legales $y$ tributarias al día.

4. Propuestos acorde a objetivos buscados.

5. Presupuesto o costos.

6. Referencias.

\section{Aspectos positivos e inconvenientes}

Por último, se le preguntó sobre cuáles podrían ser las posibles desventajas de la subcontratación, e indicó que uno de los factores de mayor influencia negativa es el poco conocimiento de la industria por parte del ente contratante, lo que puede conllevar a subcontrataciones no tan eficientes como se esperaría en un proceso externo, no conocer la cultura de 
desarrollo organizacional, y la presentación de licitaciones a un precio mayor que si se realizara con los recursos internos.

En la Tabla 1 se muestra un resumen a manera de comparación de las principales temáticas tratadas con las personas entrevistadas, a saber: cómo aplican las subcontrataciones, los procedimientos y las ventajas y desventajas.

\section{Tabla 1}

Resumen comparativo de los aspectos tratados

- Utiliza los servicios de outsourcing.

- La subcontratación la coordina la persona responsable de cada área de especialización.

\section{Empresa urbanizadora}

- Ahorro en costos que permite mejorar la rentabilidad y eficiencia

- Proceso ha evidenciado se alcanzan más fácilmente los objetivos estratégicos
- Envío de un cartel a empresas con los requisitos.

- Se requiere de al menos tres empresas.
- Flexibilidad en la rotación de colaboradores.

- Requiere de mayor supervisión de los trabajos que se ejecutan.

- Mayores controles para el cumplimiento de las tareas.

Continúa... 
- La cooperativa cree en los trabajos por outsourcing y los beneficios que generan.

- Disminución en

Coopeguanacaste R.L los costos y en los tiempos de ejecución de la obra.

- Impacto positivo en el negocio, cuando la subcontratación va de acuerdo con el plan estratégico.
- Carteles de licitación privada.

- Los requisitos de cada uno de los carteles varían de acuerdo con el alcance que se quiere cubrir con la subcontratación
- Se requiere de mayor supervisión y llevar un control estricto sobre los indicadores de costos que la organización.
- Se analiza la inversión y se relaciona con el presupuesto asignado para los rubros sujetos a subcontratarse.

- Las subcontrataciones más comunes son los procesos de capacitación, asistencia legal y fiscal, y la validación de la gestión del personal.

SherwinWilliams
- Más que un ahorro en costo, valida los procesos para los cuales son subcontratados.

- Permite alinear procedimientos y aumentar la eficiencia de las labores.

- Se subcontratan labores relacionadas con salud ocupacional, legislación laboral, aplicaciones específicas de herramientas.
- Licitaciones con esta metodología: tres cotizaciones, experiencia, condiciones legales al día, costos y referencias.
- Poco conocimiento de industria que pueda tener el contratante.

Nota: Elaboración propia con base en las entrevistas realizadas 2014 . 


\section{Conclusiones y recomendaciones}

\section{Conclusiones}

El outsourcing forma parte de la actualidad empresarial e implementarlo implica cambios en las estrategias de administración promovidas por las empresas. Hoy son muchas las organizaciones que optan por contratar servicios a un ente proveedor externo para cumplir con las demandas requeridas para su efectiva operación a un menor costo. Esto, a su vez, le permite a la empresa centrarse en su crecimiento y mejoramiento continuo. De allí que este proceso se deba realizar en función de metas específicas que tenga la organización.

La subcontratación o llamado outsourcing es una actividad de equipo; si bien es cierto, debe haber una persona responsable en la empresa de esta actividad, debe existir un equipo integrado por personas internas y externas a la organización que aseguren el buen manejo del proceso y deben involucrarse la alta gerencia y las gerencias de cada una de las áreas interesadas.

El outsourcing tiene muchas utilidades para las empresas, entre ellas:

- Reduce costos y mejora el control del gasto de operación.

- Incrementa el flujo de caja.

- Se concentra en un saber hacer sustantivo.

- Desarrolla vínculos con otras empresas de destacada experiencia y conocimiento en el área contratada.

- Aumenta la rentabilidad a la vez que incrementa la eficiencia de la operación.

- Transfiere a un tercero los riesgos de operación derivados de las contrataciones que realiza en áreas como la tecnológica, manufactura, manejo de proyecto, implementación, administración y operación de la infraestructura, entre otras. 
Entre algunas de las desventajas encontradas se destacan:

- Desarrollo de estrategias de operación basadas en un orden y control estricto para asegurar un servicio de calidad. Esto conduce a una revisión minuciosa de todas las cláusulas del contrato, lo cual genera costos añadidos.

- Incremento de la tensión para las personas de la empresa cuyo servicio se externaliza. Este puede ser mayor de lo habitual, debido a las responsabilidades que implica la inspección de funciones requeridas para asegurar la eficiencia del proveedor.

- Eventuales omisiones en los términos de la negociación que puedan ir en detrimento del costo y maximización de los beneficios.

Pérdida del control sobre la calidad del producto o servicio ahora en manos de un tercero.

- Estancamiento en lo referente a la innovación de los productos, servicios o procesos debido a que este pasa a manos de un proveedor externo.

- Paso del rol de suplidor a competidor, debido a la estrecha relación entre la empresa que contrata y el proveedor de los productos, servicios o procesos.

Por tanto, queda claro que el outsourcing debe darse en un ambiente de cooperación entre dos empresas, es decir, dos organizaciones con distintos objetivos y planteamientos estratégicos, las cuales se alían para poder compartir un mismo ideal, servir al cliente y obtener un mayor beneficio o utilidad. De manera que los términos de la contratación deben estar muy claros desde el inicio del proceso para todas las partes. 


\section{Recomendaciones}

Los objetivos que se persiguen con un proceso de outsourcing deben ser comunicados a todos los miembros de la empresa involucrados con los servicios, esto para garantizar un proceso colaborativo y consensuado.

Además de estos, otros asuntos tales como:

- Es importante que la empresa que decida aplicar el servicio de outsourcing trabaje previamente en su personal creando una cultura de actitud positiva hacia la operación de outsourcing para garantizar el éxito de su implementación.

- Entre las personas que conformen el equipo de negociación de outsourcing deben incluirse además de colaboradoras de la empresa, profesionales externos de derecho, ingeniería y especialistas según la naturaleza del servicio; su contratación no suele darse en el modelo de outsourcing.

- Es necesario realizar un análisis de los entes proveedores, tanto su solidez financiera, como experiencia en la actividad, imagen, referencias y estabilidad laboral; dado que al involucrarlos en los procesos internos, sus problemas afectarán en forma directa los de la empresa.

- Es importante destacar que entre los servicios comúnmente asociados al esquema de contratación outsourcing están aquellos pertenecientes al área financiera, contable, mercadeo, recursos humanos, administrativa, de servicios secundarios, transporte, ventas y distribución, entre otros.

- La obtención de retroalimentación por parte de empleados y clientes antes y después del outsourcing es muy valios, para comparar niveles de servicio, para lo cual se pueden utilizar encuestas, buzón de sugerencias o cualquier otra herramienta. 
- Es recomendable al iniciar la implementación del outsourcing, como estrategia de administración, desarrollar un planteamiento de forma escalonada y en fases independientes para poder ir midiendo el impacto y corregir cualquier desviación.

- Es recomendable mantener un contacto continuo con la empresa con la que se ha contratado el servicio, evaluando continuamente le funcionamiento del área en el que se presta, y comparando los resultados continuamente, para verificar si se está cumpliendo con los objetivos planteados inicialmente.

\section{Referencias}

Chacón, L. (2011). Microeconomía: De la teoría económica a la práctica empresarial. México: Pearson Education.

Cleri, C. (2007). El libro de las PYMES. Buenos Aires, Argentina: Ediciones Granica S.A.

Fórneas, J. R. (2008). Outsourcing: Saque el máximo partido de sus proveedores. España: Netbiblo.

Hofer, Ch. y Schendel, D. (2014). Strategic Management: formulation and Implementation. México: Editorial Pearson.

Koontz, H., Weihrich, H. y Cannice, M. (2012). Administración: Una perspectiva global y empresarial (14 ed.). Perú: McGraw Hill Education.

Méndez, J., (2012). La economía en la empresa en la sociedad del conocimiento. (4ta ed.) México : McGraw Hill Education.

Mondy, R. W. (2010). Administración de recursos humanos. México: Pearson.

Rodríguez, A. (2013, 16 de enero). Costa Rica se ubica como el mejor país de Latinoamérica en outsourcing. El financiero. Recuperado de http://www.elfinancierocr.com/economia-y-politica/Costa-Rica-mejorLatinoamerica-outsourcing_0_228577142.html

Schneider, B. (2004). Outsourcing. México: Grupo Editorial Norma. 